\title{
Sensitive quantitative analysis of psilocin and psilocybin in hair samples from suspected users and their distribution in seized hallucinogenic mushrooms
}

\author{
Liying Zhou ${ }^{1,2} \cdot$ Ping Xiang ${ }^{2} \cdot$ Di Wen $^{3} \cdot$ Baohua Shen $^{2} \cdot \mathrm{Xin} \mathrm{Wang}^{2} \cdot \mathrm{Le} \mathrm{Li}^{1,2} \cdot \mathrm{Hongxiao} \mathrm{Deng}^{2} \cdot \mathrm{Hang} \mathrm{Chen}^{2}$. \\ Hui Yan ${ }^{2} \cdot$ Min Shen $^{2} \cdot$ Yan Shi ${ }^{2}\left(\mathbb{C}^{\circ} \cdot\right.$ Wanhui Liu $^{1}$
}

Received: 28 October 2020 / Accepted: 17 December 2020 / Published online: 2 February 2021

(c) The Author(s) 2021

\begin{abstract}
Purpose In this study, we developed a very sensitive method for quantitative analysis of psilocin and psilocybin in hair samples of magic mushroom consumers.

Methods The analyses were performed with pretreatments of samples, followed by ultra-high pressure liquid chromatography (LC) connected to a Q-Trap type tandem mass spectrometry (MS/MS). For LC, mobile phase (A) consisted of $0.1 \%$ formic acid in water, and mobile phase (B) was acetonitrile for gradient elution using a Acquity ${ }^{\mathrm{TM}}$ UPLC HSS T3 column. For MS/ MS, electrospray ionization measurements in positive selected reaction monitoring mode were used.

Results The calibration curves were linear from 5 to $500 \mathrm{pg} / \mathrm{mg}(r>0.99)$ and no selectivity problems occurred. The limit of detection was $1 \mathrm{pg} / \mathrm{mg}$, and the lower limit of quantitation was $5 \mathrm{pg} / \mathrm{mg}$. The ranges of the matrix effects and recovery rates were $90.4-107 \%$ and $76.0-102 \%$, respectively.

Conclusions The concentrations of psilocin in two authentic hair were 161 and $150 \mathrm{pg} / \mathrm{mg}$, respectively, and psilocybin was not detected from both samples. This method was also used to analyze the distribution of psilocin and psilocybin in seven hallucinogenic mushrooms. To our knowledge, this is the first demonstration of psilocin concentrations in hair samples of hallucinogenic mushroom consumers, and also our method is most sensitive for quantitative analysis of psilocin and psilocybin in hair samples.
\end{abstract}

Keywords Psilocin $\cdot$ Psilocybin $\cdot$ Hair analysis $\cdot$ Hallucinogenic mushrooms $\cdot$ UHPLC-MS/MS

\section{Introduction}

Psilocin and psilocybin are the main hallucinogenic compounds of mushrooms such as Psilocybe, Panaeolus and Conocybe mushrooms that are commonly known as "magic mushrooms" or "hallucinogenic mushrooms". These compounds belong to the new psychoactive substance class of tryptamines and are traditional natural neuropsychiatric drugs that can cause hallucinogenic effects after taking them, producing time and space deformation and hallucinations. Hallucinogenic mushrooms were first used by North American Indians in religious ceremonies, and they were gradually abused because they can make people feel happy $[1,2]$. In recent years, cases of the abuse of hallucinogenic mushrooms have been reported in many countries. Abusers usually abuse the dried products of hallucinogenic mushrooms, or grind the hallucinogenic 
mushrooms into powder and consume the powder in the capsule form. Additionally, psilocin and psilocybin were listed in Category I of the Control Catalogue of Psychotropic Drugs of China in 2013. Long-term use of such hallucinogenic mushrooms will cause people to have adverse mental symptoms, even addiction, and these individuals will be unable to extricate themselves $[3,4]$.

Since psilocin is easily oxidized during sample preparation and/or psilocybin is dephosphorylated into psilocin in the human body by alkaline phosphatase, it is quite difficult to accurately and reliably determine trace amounts of psilocin and psilocybin in biological samples [4]. Several methods have been developed for the identification of psilocin and psilocybin in hallucinogenic mushroom, urine, and blood samples [5-11]. Many forensic toxicology laboratories use gas chromatography (GC) or GC-mass spectrometry (MS) to detect psilocin and psilocybin in hallucinogenic mushroom [12], blood [13] or urine [14] samples after derivatization. High-performance liquid chromatography (HPLC)-ultraviolet (UV) detection has also been used to analyse psilocin and psilocybin in hallucinogenic mushrooms [7, 15] and plasma [9]. In addition, some validated liquid chromatography (LC)-MS/MS methods were used to analyse psilocin and psilocybin in hallucinogenic mushroom [16], urine [17] and blood [6, 11] samples. Given that the metabolism of psilocin is rapid and unstable under the influence of light and air, the analysis of psilocin in body fluids is relatively challenging [18]. There is only one report describing the quantification of psilocybin $(0.8 \mathrm{ng} / \mathrm{mg})$ in the hair of a Psilocybe consumers [19]. However, there are no reports on the analysis of psilocin in authentic human hair.

This study proposes a sensitive and highly specific ultrahigh-performance liquid chromatography (UHPLC)-MS/ MS method for quantitative analysis of psilocin and psilocybin in seized hallucinogenic mushrooms and hair from suspected users (Fig. 1).

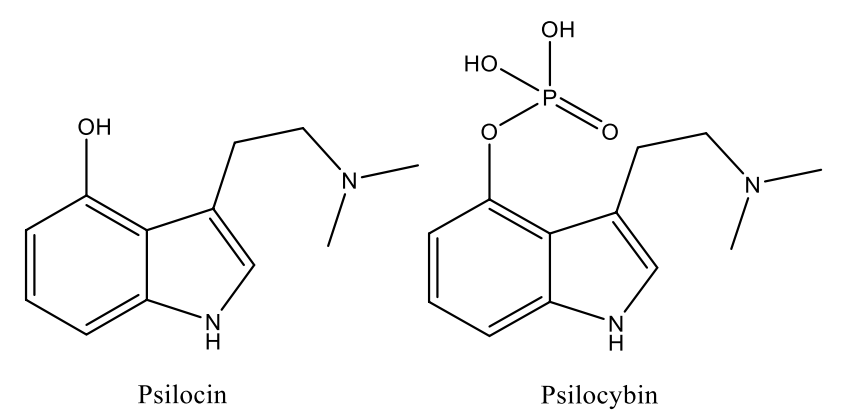

Fig. 1 Structure of psilocin and psilocybin

\section{Materials and methods}

\section{Chemicals and reagents}

Psilocin $(1 \mathrm{mg} / \mathrm{mL})$, psilocybin $(1 \mathrm{mg} / \mathrm{mL})$, and the internal standards (ISs) psilocin- $d_{10}$ and psilocybin- $d_{4}(100 \mu \mathrm{g} / \mathrm{mL})$ were purchased from Cerilliant (Round Rock, TX, USA); HPLC-grade methanol and acetonitrile from Sigma-Aldrich (St. Louis, MO, USA); analytical-grade acetone from Shanghai Lingfeng Chemical Reagent Co. (Shanghai, China); formic acid (98\%) from Fluka (Buchs, Switzerland); and deionized water was produced by an in-house Milli-Q water system (Millipore, Burlington, MA, USA).

\section{In vitro and in vivo samples}

The seven suspicious mushrooms used to identify psilocin and psilocybin were obtained from the police and customs with permission.

Human blank (drug-free) hair came from colleagues who had no history of drug use [20]. Authentic hair samples used to analyse psilocin and psilocybin were obtained from suspected users. All hair samples were collected with written informed consent.

\section{Preparation of calibrators and quality control sample}

A mixed stock solution containing psilocin and psilocybin was diluted with methanol to reach each concentration of $10 \mu \mathrm{g} / \mathrm{mL}$. From this solution, working solutions with concentrations at 1000,100 and $10 \mathrm{ng} / \mathrm{mL}$ in methanol were prepared. The IS stock solution was prepared by mixing 100 $\mu \mathrm{L}$ of $100 \mu \mathrm{g} / \mathrm{mL}$ psilocin- $d_{10}$ or psilocybin- $d_{4}$ in methanol. The IS stock solution was diluted with methanol to provide an IS working solution at a concentration of $100 \mathrm{ng} / \mathrm{mL}$.

Calibration standards were prepared by spiking blank hair with the working solutions to obtain final concentrations of $5,10,50,100,200,400$ and $500 \mathrm{pg} / \mathrm{mg}$ for psilocin and psilocybin. Low (5 and $50 \mathrm{pg} / \mathrm{mg}$ ), medium (100 pg/mg) and high $(400 \mathrm{pg} / \mathrm{mg})$ concentrations were used for quality control (QC) samples. All standard stock solutions, working solutions, and QC samples were stored at $-20^{\circ} \mathrm{C}$.

\section{Solid sample preparation}

An appropriate amount of the suspicious mushroom was cut with scissors. Twenty milligrams of each mushrooms was weighed and placed in a $2 \mathrm{~mL}$ grinding tube, and $1 \mathrm{~mL}$ of extraction solvent (methanol) was added. The sample was placed in a Shanghai Jing Xin JXFSPRP-CLN freeze 
grinding machine (Shanghai Jing Xin Industrial Development Co., Ltd., Shanghai, China), and it was ground twice at a temperature below $4{ }^{\circ} \mathrm{C}$. The parameters were set as follows: speed, $2500 \mathrm{rpm}$; number of runs, 15 times; interval time, $60 \mathrm{~s}$; and grinding, $18 \mathrm{~m} / \mathrm{s}$. Then, the sample was centrifuged at $13,500 \times g$ for $5 \mathrm{~min}$. A total of $200 \mu \mathrm{L}$ of the supernatant was placed directly into an autosampler vial for GC-MS analysis. Subsequently, the supernatant was diluted 10,000 times with $0.1 \%$ formic acid in water and directly analysed by LC-MS/MS.

Hair samples were consecutively rinsed three times with acetone. After drying at room temperature, the hair was cut into 1-2 $\mathrm{mm}$ pieces with scissors. Subsequently, $20 \mathrm{mg}$ of hair was weighed and placed in a $2 \mathrm{~mL}$ grinding tube, and $0.5 \mathrm{~mL}$ of extraction solvent (composed of $0.1 \%$ formic acid-water containing $1 \mathrm{ng}$ of ISs) was added. Then, the sample was placed in the Shanghai Jing Xin JXFSPRP-CLN freeze grinding machine for grinding under the same condition as that for mushroom. The sample was centrifuged twice at $14,100 \times g$ for $3 \mathrm{~min}$ each time. Finally, $200 \mu \mathrm{L}$ of the supernatant was pipetted into the autosampler vial, and $5 \mu \mathrm{L}$ was injected into the $\mathrm{LC}-\mathrm{MS} / \mathrm{MS}$.

\section{GC-MS}

GC-MS analysis was performed using an Agilent 7890B GC/5977A MS (Agilent Technologies, Santa Clara, CA, USA). An Agilent 7890B gas chromatograph equipped with an HP-5MS analytical column $(30 \mathrm{~m} \times 0.25 \mathrm{~mm}$, film thickness $0.25 \mathrm{~mm}$; Agilent Technologies) and helium as the carrier gas $(1.0 \mathrm{~mL} / \mathrm{min})$ was employed. The $\mathrm{GC}$ was coupled to an HP5977A mass detector in electron ionization (EI) mode at $70 \mathrm{eV}$. The temperature was held at $100{ }^{\circ} \mathrm{C}$ for $1.5 \mathrm{~min}$, increased at $25{ }^{\circ} \mathrm{C} / \mathrm{min}$ to $280^{\circ} \mathrm{C}$, and then held for $15 \mathrm{~min}$. The ion source and quadrupole temperatures were 230 and $150{ }^{\circ} \mathrm{C}$, respectively. The inlet and interface temperatures were 250 and $280^{\circ} \mathrm{C}$, respectively. The scanning range was $\mathrm{m} / \mathrm{z} 40-400$ at $0-3 \mathrm{~min}$, and the scanning range was $\mathrm{m} / \mathrm{z}$ $50-550$ at $4-6 \mathrm{~min}$.

\section{LC-MS/MS}

LC-MS/MS analysis was performed using an ultra-high pressure Acquity ${ }^{\mathrm{TM}}$ Ultra Performance LC system (Waters Corporation, Milford, MA, USA) and a Sciex 6500 Plus Q-trap ${ }^{\mathrm{TM}}$ quadrupole mass spectrometer fitted with a Turbolon Spray interface (AB Sciex, Framingham, MA, USA). The data were analysed using MultiQuant 3.0.2 software (AB Sciex).

The analytes were separated on a Waters Acquity TM UPLC HSS T3 column $(100 \times 2.1 \mathrm{~mm}$ i.d., partical size
Table 1 Progress of gradient elution for the ultra-high performance liquid chromatography-tandem mass spectrometry (UHPLC-MS/ MS)

\begin{tabular}{lcc}
\hline Time $(\mathrm{min})$ & Solvent A $(\%)$ & Solvent B (\%) \\
\hline Initial & 95.0 & 5.0 \\
2.00 & 95.0 & 5.0 \\
6.00 & 5.0 & 95.0 \\
7.00 & 5.0 & 95.0 \\
7.01 & 95.0 & 5.0 \\
8.00 & 95.0 & 5.0 \\
\hline
\end{tabular}

Table 2 Multiple reaction monitoring (MRM) transitions and retention times for the analytes and internal standards (ISs) for UHPLCMS/MS

\begin{tabular}{lcclll}
\hline Analyte & $m / z$ Q1 & $m / z$ Q3 & DP $(\mathrm{V})$ & $\mathrm{CE}(\mathrm{eV})$ & $\mathrm{RT}(\mathrm{min})$ \\
\hline Psilocin & 205.3 & 160.2 & 45 & 25 & 4.04 \\
& & $58.0^{\mathrm{a}}$ & 45 & 35 & 4.04 \\
Psilocin- $d_{10}$ & 215.3 & 66.1 & 45 & 36 & 4.05 \\
& & $164.1^{\mathrm{a}}$ & 45 & 25 & 4.05 \\
Psilocybin & 285.2 & 160.2 & 45 & 24 & 2.31 \\
& & $205.2^{\mathrm{a}}$ & 45 & 42 & 2.31 \\
Psilocybin- $d_{4}$ & 215.3 & 66.1 & 45 & 36 & 2.32 \\
& & $164.1^{\mathrm{a}}$ & 45 & 25 & 2.32 \\
\hline
\end{tabular}

$D P$ decluster potential, $C E$ collision energy, $R T$ retention time

${ }^{\mathrm{a}}$ Quantifier ions

$1.8 \mu \mathrm{m})$ using linear gradient elution. Mobile phase A consisted of $0.1 \%$ formic acid in water. Mobile phase B was acetonitrile. The gradient was programmed as shown in Table 1. The flow rate was set to $0.2 \mathrm{~mL} / \mathrm{min}$. The total running time was $8 \mathrm{~min}$, and the injection volume was 5 $\mu \mathrm{L}$. The temperature of the autosampler was maintained at $4{ }^{\circ} \mathrm{C}$.

The mass spectrometer was operated with positive electrospray ionization and multiple reaction monitoring (MRM) mode. The mass spectrometric parameters were set as follows: ion source temperature, $500{ }^{\circ} \mathrm{C}$; curtain gas, 20 psi (nitrogen); collision cell exit potential, $9 \mathrm{~V}$; ion source gas 1, $30 \mathrm{psi}$; ion source gas 2, $30 \mathrm{psi}$; entrance potential, $10 \mathrm{~V}$; ion spray voltage, $5000 \mathrm{~V}$; and collision activation dissociation gas, low. The MRM transitions and retention times are shown in Table 2.

\section{Method validation}

The method was validated according to the methodology validation guidelines [21, 22], including selectivity, linearity, limit of detection (LOD), lower limit of quantification (LLOQ), accuracy, precision, matrix effect and recovery. 


\section{Selectivity}

The selectivity was evaluated by analyzing hair samples from 10 different sources for the potential endogenous compounds in the hair or ISs interfering with the detection of analytes.

\section{Linearity, LOD, and LLOQ}

Calibrators were prepared at concentrations of 5, 10, 50, $100,200,400$ and $500 \mathrm{pg} / \mathrm{mg}$ for psilocin and psilocybin. Linearity was determined by analyzing and processing seven sets of calibrators and generating calibration curves with $1 / x$ weighting, and two independent analyses were performed for each set. The LOD and LLOQ were evaluated by spiked blank hair samples with decreasing concentrations of the analytes. The LOD was determined as the lowest analyte concentration with a signal-to-noise $(S / N)$ ratio of at least 3 . The LLOQ was defined as the lowest concentration of linearity with an $S / N \geq 10$ and that had acceptable precision and accuracy.

\section{Accuracy and precision}

Accuracy and precision were studied for hair samples by measuring six replicates at the LLOQ and QC samples at 5, 10,100 and $400 \mathrm{pg} / \mathrm{mg}$. The intraday and interday precisions were evaluated by the percentage coefficient of variation (\% $\mathrm{CV})$. The accuracy was assessed based on the percentage ratio of the measured nominal concentration (mean of measured/nominal $\times 100$ ). The precision should not exceed $\pm 15 \%$ for the low, medium and high concentrations, except for the LLOQ, which should not exceed $\pm 20 \%$. The accuracy should be within the range of $85-115 \%$ for the low, medium and high concentrations, except for the LLOQ, which should not be out of range of $80-120 \%$.

\section{Matrix effect and recovery}

The matrix effect and recovery were evaluated according to the method proposed by Matuszewski et al. [23] at 10, 100, and $400 \mathrm{pg} / \mathrm{mg}$ with 6 replicates. The samples are divided into three groups: pre-extraction spiked samples, postextraction spiked samples and standard solutions in $0.1 \%$ formic acid in water (neat sample). The matrix effect value is the peak area of post-extraction spiked samples divided by the peak area of the standard solution in $0.1 \%$ formic acid in water. The recovery is calculated as the peak area of pre-extraction-spiked samples divided by the peak area of post-extraction-spiked samples.

\section{Results}

\section{Selection of the instrument}

In our forensic toxicology laboratory, in vitro samples are usually analysed by GC-MS technology. We used GC-MS technology to analyse psilocin and psilocybin in hallucinogenic mushrooms and found that both psilocin and psilocybin had chromatographic peaks at 8.454 and $8.455 \mathrm{~min}$, respectively (Fig. 2).Then, the GC-MS technique was used to analyze the $10 \mu \mathrm{g} / \mathrm{mL}$ standard solutions of psilocin and psilocybin, respectively, and the chromatographic peaks appearing at $8.454 \mathrm{~min}$. The analysis showed that the chromatographic peak appeared at 8.454 min was only psilocin. According to relevant literature reports, psilocybin was prone to thermal decomposition and decarboxylation to form psilocin without derivatization under GC conditions [12-14]. Therefore, the GC-MS method cannot distinguish between psilocin and psilocybin without derivatization. In addition, LC-MS and LC-MS/MS have the advantages of analyzing compounds that are easily decomposed by heat and are inherently highly sensitive [24] Therefore, we chose to use LC-MS/MS technology, which did not require derivatization to analyse psilocin and psilocybin in hallucinogenic mushrooms. The chromatographic separation conditions were optimized to obtain good resolution and peak shape. After optimization, $0.1 \%$ formic acid in water (A) and acetonitrile (B) were selected as the mobile phase; good resolution and good peak shape were obtained, and a gradient elution procedure was adopted.

\section{Optimization of sample preparation}

Psilocin and psilocybin are unstable in solution, and they are completely inactivated after storage in methanol at room temperature for several months. According to relevant literature reports, psilocin and psilocybin were stable in acidic solutions [17]. There have been some reports in the literature about the use of acidic solvents to extract tryptamine compounds [25]. Therefore, we optimized four extraction solvents: $1 \%$ formic acid-methanol, $1 \%$ formic acid-water, $0.5 \%$ formic acid-water, and $0.1 \%$ formic acidwater. When methanol was used as the extraction solvent, tailing peaks tended to appear in the chromatogram. By comparing the extraction recovery and matrix effect of different extraction solvents, the results showed that $0.1 \%$ formic acid-water was the best extraction solvent for hair samples. Moreover, Martin et al. [25] used hydrochloric acid-methanol extraction and sonication for $6 \mathrm{~h}$ to extract psilocin from $50 \mathrm{mg}$ of hair and performed extract cleanup by solid-phase extraction using a mixed-mode cation 


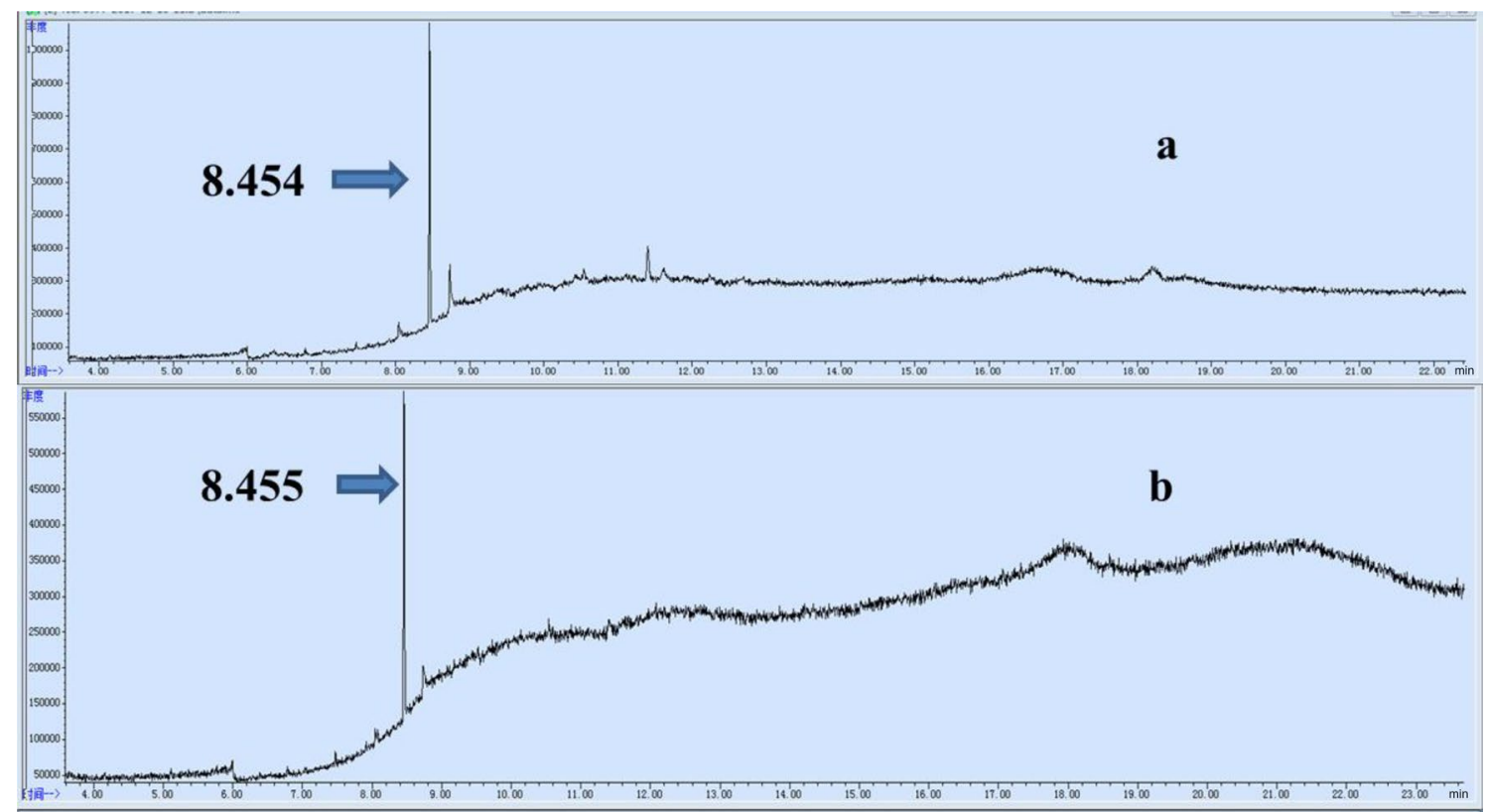

Fig. 2 Selected ion monitoring chromatograms of psilocin (a) and psilocybin (b) in hallucinogenic mushrooms were analysed by gas chromatography-mass spectrometry technology

exchanger [20]. We used a Shanghai Jing Xin JXFSPRPCLN freeze grinding machine to grind approximately $20 \mathrm{mg}$ of human hair below $4{ }^{\circ} \mathrm{C}$ and utilized $0.1 \%$ formic acid-water to extract psilocin and psilocybin. The cryo-grinding method ensured the stability of thermally unstable compounds, and at the same time, it could pulverize the hair completely so that the drug in the hair was released relatively completely, and the operation was simple and timesaving. In addition, the method developed in this study provided higher sensitivity and a lower limit of quantification than those of the previous method $[6,11$, $16,17]$.

\section{Method validation}

The selectivity of the method was evaluated using 10 blank hair matrix samples and internal standards from different sources, and no endogenous interference peaks or interference from the ISs with the analytes were observed.

The linearity, LOD, and LLOQ of psilocin and psilocybin in human hair are listed in Table 3 . The linear range was $5-500 \mathrm{pg} / \mathrm{mg}$, and the correlation coefficient $(r)$ was higher than 0.99 . The LOD value of psilocin and psilocybin was $1 \mathrm{pg} / \mathrm{mg}$, and the LLOQ value was $5 \mathrm{pg} / \mathrm{mg}$ for human hair samples. The LOD and LLOQ values of this method were lower than those of previous studies (LOD $=10 \mathrm{pg} / \mathrm{mg}, \mathrm{LLOQ}=22 \mathrm{pg} / \mathrm{mg}$ ) [25], which means that this method has sensitivity higher than any analytical method for psilocin and psilocybin in human hair.

The accuracy and intraday and interday precisions of psilocin and psilocybin at the four levels are shown in Table 4 . The accuracy and intraday and interday precision values met the acceptable criteria. The intraday precisions ranged from 6.1 to $10.5 \%$, and the interday precisions ranged from 2.2 to $6.7 \%$. The accuracy was between 93.6 and $112 \%$.

The results of the matrix effect and recovery analyses are also shown in Table 4. The matrix effects of psilocin and psilocybin ranged from 90.4 to $107 \%$ at 10 (low), 100 (medium), and $400 \mathrm{pg} / \mathrm{mg}$ (high) (\% RSD $\leq 20 \%)$. The results indicated that the analytes did not exhibit significant ion suppression or ion enhancement in this method. The recoveries of psilocin and psilocybin were within a range of $76.0-102 \%$.
Table 3 Linearity, limit of detection (LOD) and lower limit of quantification (LLOQ) for psilocin and psilocybin in human hair obtained by the UHPLC-MS/MS

\begin{tabular}{llllll}
\hline Analyte & Linearity $(\mathrm{pg} / \mathrm{mg})$ & Regression equation & $\begin{array}{l}\text { Correlation } \\
\text { coefficient }(r)\end{array}$ & $\begin{array}{l}\text { LOD }(\mathrm{pg} / \\
\mathrm{mg})\end{array}$ & $\begin{array}{l}\text { LLOQ } \\
(\mathrm{pg} / \\
\mathrm{mg})\end{array}$ \\
\hline Psilocin & $5-500$ & $\mathrm{y}=0.3652 x-0.0003161$ & 0.994 & 1 & 5 \\
Psilocybin & $5-500$ & $\mathrm{y}=1.99 x-0.00371$ & 0.992 & 1 & 5 \\
\hline
\end{tabular}


Table 4 Accuracies, precisions, matrix effects and recoveries for psilocin and psilocybin in human hair measured by the UHPLC-MS/MS

\begin{tabular}{|c|c|c|c|c|c|c|}
\hline \multirow[t]{2}{*}{ Analyte } & \multirow{2}{*}{$\begin{array}{l}\text { Concentration } \\
(\mathrm{pg} / \mathrm{mg})\end{array}$} & \multirow[t]{2}{*}{ Accuracy (\%) } & \multicolumn{2}{|l|}{ Precision (\%) } & \multirow{2}{*}{$\begin{array}{l}\text { Matrix effect }(\%) \\
(n=6)\end{array}$} & \multirow{2}{*}{$\begin{array}{l}\text { Recovery }(\%) \\
(n=6)\end{array}$} \\
\hline & & & Intraday $(n=6)$ & Interday $(n=6)$ & & \\
\hline \multirow[t]{4}{*}{ Psilocin } & 5 & 108 & 6.1 & 2.2 & - & - \\
\hline & 10 & 96.4 & 10.5 & 6.7 & 93.1 & 87.1 \\
\hline & 100 & 97.5 & 9.1 & 3.9 & 90.8 & 76.0 \\
\hline & 400 & 106 & 9.2 & 5.5 & 103 & 102 \\
\hline \multirow[t]{4}{*}{ Psilocybin } & 5 & 112 & 9.5 & 2.8 & - & - \\
\hline & 10 & 93.6 & 8.3 & 4.2 & 90.4 & 88.2 \\
\hline & 100 & 94.6 & 9.3 & 4.0 & 107 & 92.0 \\
\hline & 400 & 110 & 9.8 & 4.0 & 104 & 101 \\
\hline
\end{tabular}

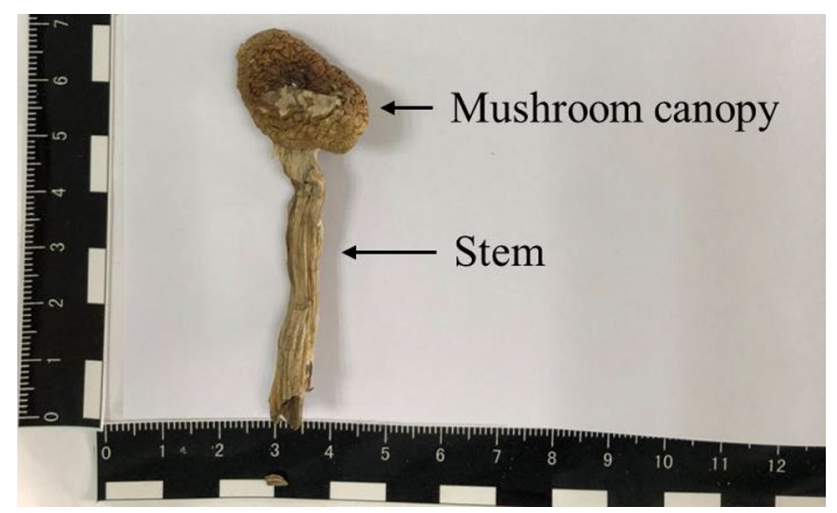

Fig. 3 An example of the seized hallucinogenic mushrooms containing psilocin and psilocybin

\section{Application of the method}

The UHPLC-MS/MS method proposed in this study was used to successfully detect and quantitatively analyze psilocin and psilocybin in seized hallucinogenic mushrooms and two authentic human hair samples.

\section{Quantitative analysis of psilocin and psilocybin in seized hallucinogenic mushrooms}

In recent years, hallucinogenic mushrooms have often been sold in their dried form, ground powder or capsules. At present, there have been some reports in the literature using LC-MS or GC-MS to analyse the content and distribution of psilocin and psilocybin in hallucinogenic mushrooms [15]. The psilocin content in six hallucinogenic mushrooms was determined to be lower than $12.67 \mathrm{ng} / \mathrm{mg}$ by HPLC combined with the fluorescence derivatization method [4]. The police seized a batch of suspicious mushrooms during a customs security check and sent them to our forensic toxicology laboratory for testing (Fig. 3). Seven suspicious mushrooms and $50 \mathrm{ng} / \mathrm{mL}$ mixed standard dilutions of psilocin and psilocybin were separately analysed by LC-MS/MS.
Both the suspicious mushroom and the standard samples showed chromatographic peaks at $4.04 \mathrm{~min}$ and $2.29 \mathrm{~min}$, indicating that the suspicious mushroom contained psilocin and psilocybin (Fig. 4). The quantitative analysis results of psilocin and psilocybin in 7 suspicious mushrooms are shown in Table 5. The results showed that the contents of psilocin and psilocybin in the canopy of hallucinogenic mushrooms were higher than those in the stem (Table 5).

\section{Quantitative analysis of psilocin and psilocybin in hair samples of suspected users}

A 25-year-old man (case 1) was driving home, speeding, and laughing. The driver was stopped by the police, and the remaining suspicious poisonous mushrooms were seized from the car. It was found that the driver's ability to respond was low; his eyes were dull and red, and his standing was unstable. He was brought to the hospital immediately and recovered. The concentration of psilocin detected in his hair was $161 \mathrm{pg} / \mathrm{mg}$, and psilocybin was not detected.

A 31-year-old male (case 2) with a history of drug use went to a party at an entertainment venue. At the party, he drank and smoked suspicious powder. After $30 \mathrm{~min}$, he experienced hallucinations, felt his surroundings moved, and laughed. After receiving the report, the police seized the suspicious powder at the scene and sent the man to the hospital. The suspicious powder contained psilocin and psilocybin. The concentration of psilocin detected in his hair was $150 \mathrm{pg} / \mathrm{mg}$, and psilocybin was not detected (Fig. 5).

\section{Discussion}

It has been reported that in the human body, psilocybin is rapidly dephosphorylated to form psilocin, and most psilocin is combined with glucuronic acid and excreted in urine. Small amounts of psilocin and psilocybin in hallucinogenic mushrooms can induce ecstasy, but overdose or administration with additional drugs can be fatal [9]. 

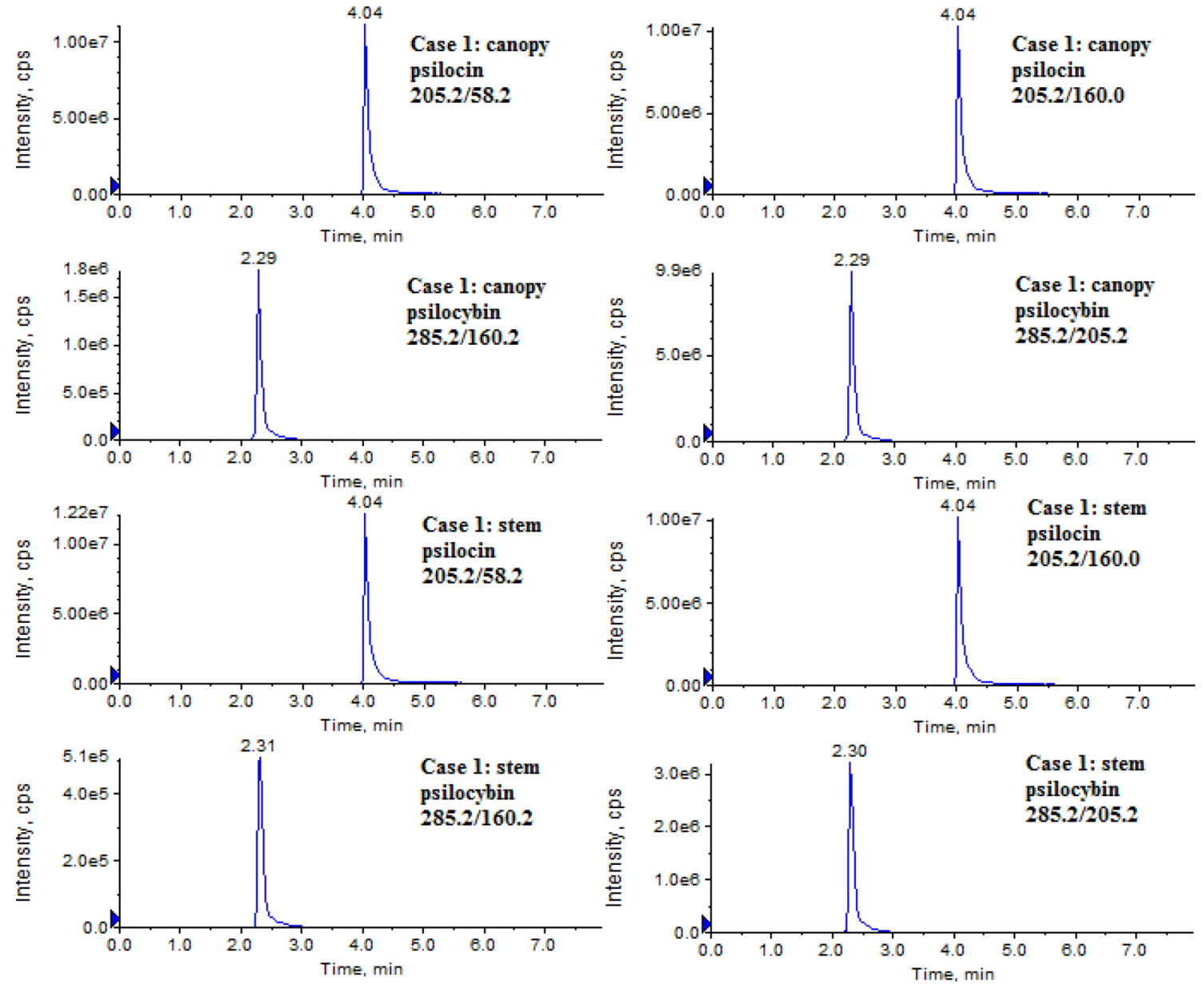

Fig. 4 Multiple reaction monitoring (MRM) chromatogram for psilocin and psilocybin in the canopy and stem

Table 5 Contents of psilocin and psilocybin in seven suspicious mushrooms (ng/mg)

\begin{tabular}{|c|c|c|c|c|c|c|}
\hline \multirow[t]{2}{*}{ Case } & \multicolumn{2}{|c|}{ Mushroom canopy } & \multirow[t]{2}{*}{ Ratio } & \multicolumn{2}{|l|}{ Stem } & \multirow[t]{2}{*}{ Ratic } \\
\hline & $\begin{array}{l}\text { Psilocybin (ng/ } \\
\mathrm{mg})\end{array}$ & $\begin{array}{l}\text { Psilocin (ng/ } \\
\mathrm{mg})\end{array}$ & & $\begin{array}{l}\text { Psilocybin (ng/ } \\
\mathrm{mg})\end{array}$ & Psilocin (ng/mg) & \\
\hline 1 & 5.49 & 6.24 & 0.88 & 4.11 & 5.95 & 0.69 \\
\hline 2 & 4.77 & 5.39 & 0.88 & 1.18 & 2.70 & 0.44 \\
\hline 3 & 1.32 & 9.59 & 0.14 & 1.50 & 4.39 & 0.34 \\
\hline 4 & 1.22 & 3.03 & 0.40 & 0.755 & 1.89 & 0.40 \\
\hline 5 & 1.42 & 14.9 & 0.10 & 0.164 & 0.883 & 0.19 \\
\hline 6 & 1.22 & 7.50 & 0.16 & 0.033 & 0.837 & 0.04 \\
\hline 7 & 0.62 & 19.6 & 0.03 & 0.296 & 3.28 & 0.09 \\
\hline
\end{tabular}

Sticht and Käferstein [13] used GC-MS and silylation to detect psilocin in urine and serum and found that psilocin mostly existed in the conjugated form, and the concentration of psilocin detected in urine and blood was 0.018 and $0.052 \mathrm{mg} / \mathrm{L}$, respectively. However, Tiscione and Miller [26] analysed the concentration of free psilocin in urine samples to be $0.23 \mathrm{mg} / \mathrm{L}$ by GC-MS. The analysis of psilocin in body fluids is relatively challenging because psilocin is rapidly metabolized and unstable under the influence of light and air [18].

As to psilocin concentration in human hair samples collected from magic mushroom consumers, no report on its detection appeared. Only one report on the quantitative detection of psilocybin from hair $(0.8 \mathrm{ng} / \mathrm{mg})$ of magic mushroom consumer appeared, but psilocin was not detected [19]. Therefore, this report is the first to succeed 

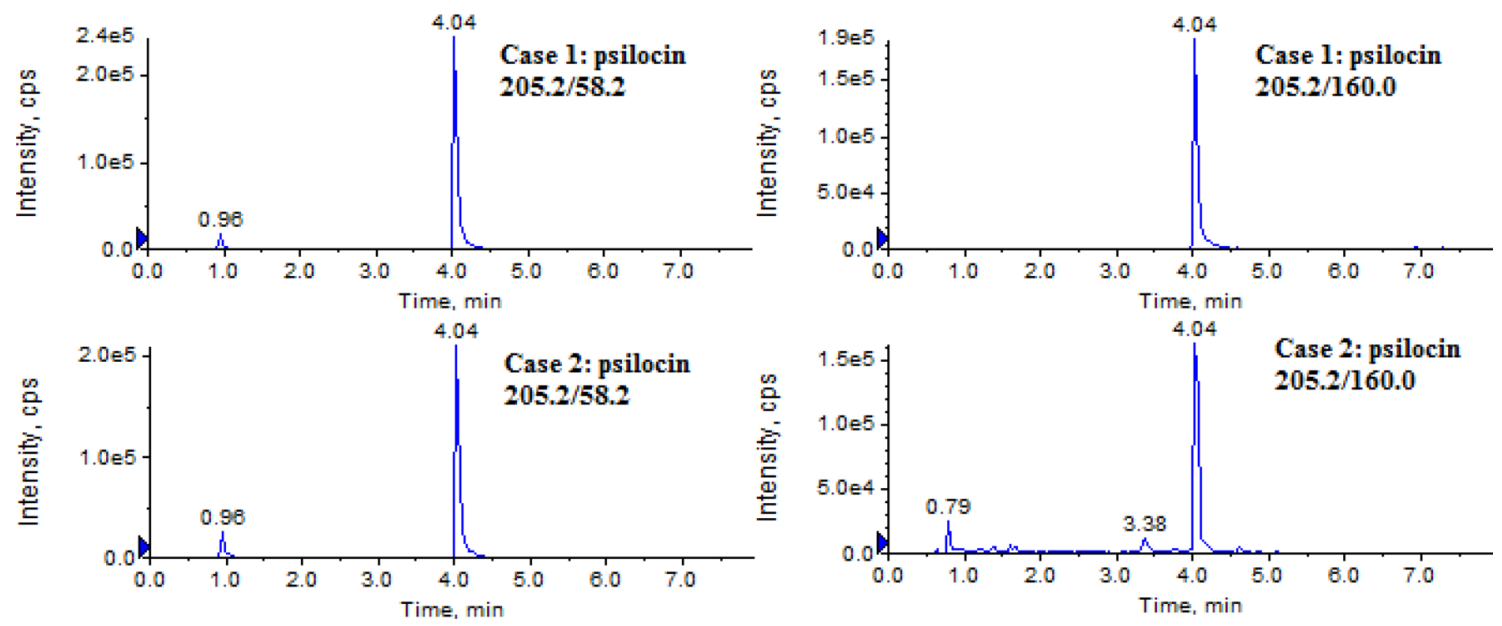

Fig. 5 MRM chromatogram for psilocin in the hair samples of magic mushrooms consumers

quantitative detection of psilocin from authentic human hair.

The reason why the detection of psilocin in hair specimens of magic mushrooms should be pointed out. First, we need the most modern Q-trap type MS instrument, which seems to be extraordinarily sensitive, coupled to a special high-quality UHPLC system. Second, the fresh hair specimens were cut off just before the beginning the analysis, followed by pulverizing to fine powder. Third, special care was taken to prevent the specimens from the decomposition of psilocin by light [17] at low temperature, using alkaline $\mathrm{pH}$ solution during the pretreatment. As results, the concentrations of psilocin in hair specimens from two subjects were 161 and $150 \mathrm{pg} / \mathrm{mg}$, respectively. Psilocybin could not be detected from both hair specimens, despite that the LOD and LLOQ were as low as 1 and $5 \mathrm{pg} / \mathrm{mg}$, respectively, which seems due to metabolism of psilocybin to psilocin in vivo in the humans.

At the next study, we have studied the distribution of psilocin and psilocybin in the canopies, stems and hyphae of hallucinogenic mushrooms themselves. Since such studies had been well conducted $[12,15,16]$, we compared our results with the published data as shown in Tables 5 and 6 . Our results and those in the previous studies [7, 12] equally indicated that the concentration of psilocybin in the stems and hyphae of hallucinogenic mushrooms are lower than in the canopy. We examined the ratio of psilocybin to psilocin in the canopies and stems of hallucinogenic mushrooms, and the results are listed in Table 5. The ratio of psilocybin to psilocin in the canopies of the 7 samples ranged from 0.03 to 0.88 , while the ratio of psilocybin to psilocin in the stems ranged from 0.04 to 0.69 . The results show that the psilocin content is higher than the psilocybin content in the canopies and stems of hallucinogenic mushrooms. The data in the previously published literature showed that the contents of psilocybin in the canopies and stems of hallucinogenic mushrooms were higher than those of psilocin, which was exactly the opposite of our experimental results $[12,15,16]$. However, the literature published by Tsujikawa et al. [27]

Table 6 Data on the contents of psilocin and psilocybin in hallucinogenic mushrooms reported in the relevant literatures

\begin{tabular}{|c|c|c|c|c|c|}
\hline Case & Species & Psilocybin (ng/mg) & Psilocin (ng/mg) & Ratio & References \\
\hline 1 & Psilocybe srgentipes & 3.8 & 0.69 & 5.51 & {$[16]$} \\
\hline 2 & Psilocybe srgentipes & 3.2 & 0.60 & 5.33 & \\
\hline 3 & Psilocybe subcubensis & 1.5 & 1.0 & 1.5 & \\
\hline 4 & Unknown & 0.18 & 1.4 & 0.13 & \\
\hline 5 & Psilocybe cubensis (dried) & 11.51 & 1.26 & 9.17 & {$[15]$} \\
\hline 6 & Psilocybe cubensis (fresh) & 7.92 & 0.66 & 12.00 & \\
\hline 7 & Psilocybe cubensis (grow box) & 5.09 & 1.27 & 4.01 & \\
\hline 8 & Panaeolu scyanescens (dried) & 25.14 & 11.94 & 2.10 & \\
\hline 9 & Psilocybe tampanensis (fresh) & 1.19 & 0.58 & 2.05 & \\
\hline 10 & Psilocybe subcubensis & 8.6 (canopy), 8.0 (stem) & 0.2 (canopy), 0.3 (stem) & $\begin{array}{l}43 \text { (canopy), } 26.7 \\
\quad \text { (stem) }\end{array}$ & {$[12]$} \\
\hline
\end{tabular}


showed that the contents of psilocin in the canopies and stems of Copelandia were higher than those of psilocybin (0.64-0.74\%/0.02-0.22\% (psilocin/psilocybin) in the canopies and $0.31-0.78 \% / 0.01-0.39 \%$ (psilocin/psilocybin) in the stems), which is consistent with our experimental results. Kamata et al. [16] used LC-MS/MS to analyse the concentration ranges of psilocin and psilocybin in 4 real samples, which were $0.6-1.4 \mathrm{mg} / \mathrm{g}$ and $0.18-3.8 \mathrm{mg} / \mathrm{g}$, respectively. The data previously reported in the relevant literature on the contents of psilocin and psilocybin in hallucinogenic mushrooms are summarized in Table 6.

Some drugs are rapidly metabolized in the body, and the parent drug cannot be detected in conventional biological samples such as urine but can be detected in hair [28]. Therefore, hair analysis is a useful tool for retrospective drug screening. The time and length of drug intake can be evaluated by correlating the position of the analyte in the hair sample with the hair growth rate [29].

\section{Conclusions}

This paper established a liquid chromatography-tandem mass spectrometry method to detect and quantitatively analyze psilocin and psilocybin in human hair. This method was successfully applied to analyze psilocin in two real hair samples. The concentrations of psilocin detected in the hair samples were 161 and $150 \mathrm{pg} / \mathrm{mg}$, respectively, while psilocybin was not detected. To our knowledge, this is the first demonstration of psilocin in hair specimens of hallucinogenic mushroom consumers. In addition, distribution of psilocin and psilocybin was given in seven mushroom seizure cases. The present method is most sensitive for quantitative analysis of psilocin and psilocybin in human hair among those so far reported.

Acknowledgements This study was supported by grants from Shanghai Science and Technology Commission (19DZ1200600), National Natural Science Foundation of China (81971789, 81772022), Research Institute Projects (GY2019G-4 and GY2020Z-2), Shanghai Key Laboratory of Forensic Medicine (17DZ2273200) and Shanghai Forensic Service Platform (19DZ2292700).

\section{Compliance with ethical standards}

Conflict of interest The authors declare that they have no conflict of interest.

Ethical approval This article does not contain any studies with human participants or animals performed by any of authors.

Open Access This article is licensed under a Creative Commons Attribution 4.0 International License, which permits use, sharing, adaptation, distribution and reproduction in any medium or format, as long as you give appropriate credit to the original author(s) and the source, provide a link to the Creative Commons licence, and indicate if changes were made. The images or other third party material in this article are included in the article's Creative Commons licence, unless indicated otherwise in a credit line to the material. If material is not included in the article's Creative Commons licence and your intended use is not permitted by statutory regulation or exceeds the permitted use, you will need to obtain permission directly from the copyright holder. To view a copy of this licence, visit http://creativecommons.org/licenses/by/4.0/.

\section{References}

1. Hofmann A, Brack A, Kobel H, Fery A, Ott H, Petrzilka Th, Troxler F (1959) Psilocybin und Psilocin, zwei psychotrope Wirkstoffe aus mexikanischen Rauschpilzen. Helv Chim Acta 42:1557-1572. https://doi.org/10.1002/HLCA.19590420518

2. Geschwinde Th (2007) Rauschdrogen: Marktformen und Wirkungsweisen, Sechate Aultage. Springer, Berlin

3. Hasler F, Bourquin D, Brenneisen R, Bär T, Vollenweiden FX (1997) Determination of psilocin and 4-hydroxyindole-3-acetic acid in plasma by HPLC-ECD and pharmacokinetic profiles of oral and intravenous psilocybin in man. Pharm Acta Helv 72:175184. https://doi.org/10.1016/S0031-6865(97)00014-9

4. Satio K, Toyo;oka T, Fukushima T, Kato M, Shirota O, Goda Y (2004) Determination of psilocin in magic mushrooms and rat plasma by liquid chromatography with fluorimetry and electrospray ionization mass spectrometry. Anal Chim Acta 527:149156. https://doi.org/10.1016/j.aca.2004.08.071

5. Björnstad K, Beck O, Helander A (2009) A multi-component LC-MS/MS method for detection of ten plant-derived psychoactive substances in urine. J Chromatogr B 877:1162-1168. https:// doi.org/10.1016/j.jchromb.2009.03.004

6. Kamata T, Nishikawa M, Katagi M, Tsuchihashi H (2006) Direct detection of serum psilocin glucuronide by LC/MS and LC/MS/ MS: time-courses of total and free (unconjugated) psilocin concentrations in serum specimens of a "magic mushroom" user. Forensic Toxicol 24:36-40. https://doi.org/10.1007/s11419-0060006-2 (open access article)

7. Borner S, Borenneisen R (1987) Determination of tryptamine derivatives in hallucinogenic mushrooms using high-performance liquid chromatography with photodiode array detection. J Chrotnatogr 408:402-408. https://doi.org/10.1016/S0021 $-9673(01) 81831-8$

8. Wurst M, Semerdžieva M, Vokoun J (1984) Analysis of psychotropic compounds in fungi of the genus psilocybe by reversedphase high-performance liquid chromatography. J Chromatogr 286:229-235. https://doi.org/10.1016/S0021-9673(01)99190-3

9. Chen J, Li M, Yan X, Wu E, Zhu H, Lee KJ, Chu VM, Zhan L, Lee W, Kang JS (2011) Determining the pharmacokinetics of psilocin in rat plasma using ultra-performance liquid chromatography coupled with a photodiode array detector after orally administering an extract of Gymnopilus spectabilis. J Chromatogr B 879:2669-2672. https://doi.org/10.1016/j.jchromb.2011.07.003 (open access article)

10. Martin R, Schürenkamp J, Gasse A, Pfeiffer H, Köhler H (2013) Determination of psilocin, bufotenine, LSD and its metabolites in serum, plasma and urine by SPE-LC-MS/MS. Int J Legal Med 127:593-601. https://doi.org/10.1007/s00414-012-0796-1. (open accessarticle)

11. Martin R, Schurenkamp J, Gasse A, Pfeiffer H, Köhler H (2012) A validated method for quantitation of psilocin in plasma by LC-MS/ MS and study of stability. Int J Legal Med 126:845-849. https:// doi.org/10.1007/s00414-011-0652-8. (open access article) 
12. Kellera T, Regenscheitb P, Dirnhofer R, Rücker T, Jaspers J, Kisser W (1999) Analysis of psilocybin and psilocin in Psilocybe subcubensis GUZMÁN by ion mobility spectrometry and gas chromatography-mass spectrometry. Forensic Sci Int 99:93-105. https://doi.org/10.1016/S0379-0738(98)00168-6 (open access article)

13. Sticht G, Käferstein H (2000) Detection of psilocin in body fluids. Forensic Sci Int 113:403-407. https://doi.org/10.1016/S0379 -0738(00)00213-9

14. Grieshaber AF, Moore KA, Levine B (2001) The detection of psilocin in human urine. J Forensic Sci 46:627-630. https://doi. org/10.1520/JFS15014J

15. Laussmann T, Meier-Giebing S (2010) Forensic analysis of hallucinogenic mushrooms and khat (Catha edulis Forsk) using cationexchange liquid chromatography. Forensic Sci Int 195:160-164. https://doi.org/10.1016/j.forsciint.2009.12.013

16. Kamata T, Nishikawa M, Katagi M, Tsuchihashi H (2005) Liquid chromatography-mass spectrometric and liquid chromatographytandem mass spectrometric determination of hallucinogenic indoles psilocin and psilocybin in "magic mushroom" samples. J Forensic Sci 50:336-340. https://doi.org/10.1520/JFS2004276

17. Kamata T, Nishikawa M, Katagi M, Tsuchihashi H (2003) Optimized glucuronide hydrolysis for the detection of psilocin in human urine samples. J Chromatogr B 796:421-427. https://doi. org/10.1016/j.jchromb.2003.08.030

18. Anastos N, Barnett NW, Pfeffer FM, Lewis SM (2006) Investigation into the temporal stability of aqueous standard solutions of psilocin and psilocybin using high performance liquid chromatography. Sci Justice 46:91-96. https://doi.org/10.1016/S1355 -0306(06)71579-9

19. Pichini S, Marchei E, García-Algar O, Gomez A, Di Giovannandrea R, Pacifici R (2004) Ultra-high-pressure liquid chromatography tandem mass spectrometry determination of hallucinogenic drugs in hair of psychedelic plants and mushrooms consumers. J Pharm Biomed Anal 100:284-289. https://doi.org/10.1016/j. jpba.2014.08.006

20. Dinis-Oliveira RJ, Vieira DN, Magalhāes T (2016) Guidelines for collection of biological samples for clinical and forensic toxicological analysis. Forensic Sci Res 1:42-51. https://doi. org/10.1080/20961790.2016.1271098. (open access article)

21. Peters FT, Drummer OH, Musshoff F (2007) Validation of new methods. Forensic Sci Int 165:216-224. https://doi.org/10.1016/j. forsciint.2006.05.021
22. Scientific Working Group for Forensic Toxicology (2013) Scientific Working Group for Forensic Toxicology (SWGTOX) standard practices for method validation in forensic toxicology. J Anal Toxicol 37:452-474. https://doi.org/10.1093/jat/bkt054. (open access article)

23. Matuszewski BK, Constanzer ML, Chavez-Eng CM (2003) Strategies for the assessment of matrix effect in quantitative bioanalytical methods based on HPLC-MS/MS. Anal Chem 75:3019-3030. https://doi.org/10.1021/ac020361s

24. Tomková J, Ondra P, Válka I (2015) Simultaneous determination of mushroom toxins $\alpha$-amanitin, $\beta$-amanitin and muscarine in human urine by solid-phase extraction and ultra-high-performance liquid chromatography coupled with ultra-high-resolution TOF mass spectrometry. Forensic Sci Int 251:209-213. https://doi. org/10.1016/j.forsciint.2015.04.007

25. Martin R, Schürenkamp J, Gasse A, Pfeiffer H, Köhler H (2015) Analysis of psilocin, bufotenine and LSD in hair. J Anal Toxicol 39:126-129. https://doi.org/10.1093/jat/bku141 (open access article)

26. Tiscione NB, Miller MI (2006) Psilocin identified in a DUID investigation. J Anal Toxicol 30:342-345. https://doi.org/10.1101/ gad.852400. (open access article)

27. Tsujikawa K, Kanamori T, Iwata Y, Sugita OY, Inoue RH, Kishi T (2003) Morphological and chemical analysis of magic mushrooms in Japan. Forensic Sci Int 138:85-90. https://doi.org/10.1016/j. forsciint.2003.08.009

28. Boumba VA, Di Rago M, Peka M, Drummer OH, Gerostamoulos D (2017) The analysis of 132 novel psychoactive substances in human hair using a single step extraction by tandem LC/MS. Forensic Sci Int 279:192-202. https://doi.org/10.1016/j.forsc iint.2017.08.031

29. Pragst F, Balikove MA (2006) State of the art in hair analysis for detection of drug and alcohol abuse. Clin Chim Acta 370:17-49. https://doi.org/10.1016/j.cca.2006.02.019

Publisher's Note Springer Nature remains neutral with regard to jurisdictional claims in published maps and institutional affiliations. 